\title{
Scheduling of Distributed Energy Resources as a Virtual Power Plant
}

\author{
J. Qiu$^{1}$, T.S. Brinsmead ${ }^{1}$ and L.J. Reedman ${ }^{1}$ \\ ${ }^{I}$ The Commonwealth Scientific and Industrial Research Organization (CSIRO), Mayfield West NSW 2304, \\ Australia \\ E-mail: qiujing0322@gmail.com
}

\begin{abstract}
A transactive energy system can provide an integral management scheme that facilitates power delivery with high efficiency and reliability. To close the gap between wholesale and retail markets, this paper presents a two-stage optimal scheduling model for distributed energy resources (DERs) in the form of a virtual power plant (VPP) participating in the day-ahead (DA) and real-time (RT) markets. In the first stage, the hourly scheduling strategy of the VPP is optimized, in order to maximize the total profit in the DA market. In the second stage, the outputs of the VPP are optimally adjusted, in order to minimize the imbalance cost in the RT market. The conditional-value-at-risk $(\mathrm{CVaR})$ is used to assess the risk of profit variability due to the presence of uncertainties in renewable energy outputs, market prices and energy demands. The formulated two-stage models are solved by an enhanced particle swarm optimization algorithm (PSO) and the commercial solver AMPL/IPOPT 3.8.0. In the procedures of the enhanced PSO, two particles with the lowest and highest fitness values are used as the starting points, and then the interior point method will be employed to quickly locate local optima. The population size is set at 200 , and the iteration number is set at 1000 . Simulation results show that coordinated scheduling can effectively offset the renewable energy fluctuation and mitigate the impacts of uncertainties. With the two-level scheduling, the risk exposure can be mitigated, and the cost related to the risk aversion is also effectively reduced. The paper finds that coordinated two-level DERs scheduling is a flexible risk-hedging tool that can identify optimal operation, resulting in more affordable electricity prices for end users.
\end{abstract}

Keywords: Virtual power plant, transactive energy, optimal scheduling and distributed energy resources 
Nomenclature

A. Indices

$\begin{array}{ll}i & \text { Bus } \\ k & \text { Scenario } \\ t & \text { Time }\end{array}$

\section{B. Parameters}

$a_{1}^{I L}, a_{2}^{I L}$
$a_{1}^{D G}, a_{2}^{D G}, a_{3}^{D G}$

Cost coefficients of interruptible load

Cost coefficients of

distributed non-renewable

generation

$\alpha$

$\beta^{\text {BESS }}$

Confidence level

$E_{R}^{B E S S}$

$E_{\text {Initial }}^{\text {BESS }}, E_{\text {End }}^{\text {BESS }}$

$\zeta^{+}, \zeta^{-}$

IC $C^{\text {BESS }}$

$L C N$

MUT, MDT

$\eta^{C}, \eta^{L}$

N

NT

$\Delta P_{G W}$

$P^{\text {Exch }}$

$P^{\text {BESS ,Dis }}$

$P^{B E S S, C h r}$,

$\rho^{\text {Retail }}$

$R s v$

$\operatorname{Ramp}_{D G}^{U p}$

$\operatorname{Ramp}_{D G}^{\text {Down }}$

$S U C^{D G}$,

$S D C^{D G}$

$T$

$\Delta x$

$\mu_{x}, \sigma_{x}^{2}$

$\lambda_{1}, \lambda_{2}$

$\varpi$

$\psi$

lifetime degradation

Rated energy of BESS in BESS

Investment cost of BESS

Total lifecycle of BESS limits of DG (distributed generation)

Normalization factor

RT

Wind power forecast error

Interconnection power

exchange

Retail price for customers

of DG of DG

DA

Demand or price forecast

error

(std.) of demand and price

forecast error

Beta distribution

Weighting factor of risk

Auxiliary continuous $\zeta$

Initial and final energy stored

Relative differences between

DA and RT electricity prices

Minimum up and down time

Dis/charging loss factor and

leakage loss factor of BESS

Total scheduling intervals in

Dis/charging power of BESS

System reserve requirement

Ramping up and down rates

Start-up and shut-down costs

Total scheduling intervals in

Mean and standard deviation

Positive shape parameters of

nonnegative variable
C. Variables

$C^{B E S S}$

$C^{D G}$

$C^{\operatorname{Imb}}$

$C^{I L}$

$E^{B E S S}$

$P, Q$

$P_{D}, Q_{D}$

Ratio of interruptible load

Operational cost of BESS

Cost of distributed nonrenewable generation Imbalance cost

Cost of interruptible load

Energy stored in BESS

Active and reactive power injection

Active and reactive power demand

$P_{G}, Q_{G}$

$P_{D G}$

$P^{\operatorname{Imb}}$

$P^{F c t}$

$P^{D S}$

$P^{B E S S}$

$P_{G W}, Q_{G W}$

$P^{\text {Loss }}$

$P^{I L}$

$\rho^{R+} \rho^{R-}$

$\rho^{D A}$

$S$

$S O C$

$\theta, V$

$T^{O n} T^{O f f}$

VaR

$\chi^{D G}$

$\chi^{S U}, \chi^{S D}$

Generated active and

reactive power

Active power output of DG

Active power imbalance

Forecasted power

Scheduled power

BESS power

Wind active and reactive

power

Active power loss

Interruptible load power

Regulation up and down

prices

DA electricity price

Complex power flow

State of charge

Voltage angle and

amplitude

Number of hours for which

DG has been on or off

Value at risk

Commitment status of DG

Start-up and shut-down

binary decisions of DG

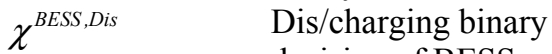

decision of BESS

$\chi^{B E S S, C h r}$

D. Sets and others

$\Omega_{G W} \quad$ Set of wind power

$\Omega_{\text {BESS }} \quad$ Set of BESS

$\Omega_{D G}$

$\Omega_{D} \quad$ Set of demand

Set of distributed non-

renewable generation

$\Omega_{K}$

Set of scenarios

$\Omega_{N}$

Set of bus

$\overline{(\bullet)},(\bullet)$

Upper and lower limits

$E(\bullet) \quad$ Expectation operator 


$\begin{array}{llll}\operatorname{Pr}(\bullet) & \text { Probability operator } & \Delta t & \text { Time factor } \\ (\bullet) & \text { Variables in RT } & & \end{array}$

\section{INTRODUCTION}

Recently, it has been proposed that transactive energy systems could be used to facilitate energy transactions between Distributed Energy Resources (DERs) and electricity markets, since more consumers are now becoming "prosumers" (i.e. both consuming and producing energy). As an agent in the retail market, a virtual power plant (VPP) aggregates the capacity of many DERs and creates an aggregate operating profile, to trade electrical energy or to provide system support services (Kardakos et al., 2016), (Pandzic et al., 2013). Unlike a microgrid, a VPP is not necessarily limited to a geographical location (Asmus, 2010); instead, it is more like a market agent representing the portfolio of DERs. Participating in electricity markets entails risks, given the many uncertainties involved, such as market prices, load forecast errors and wind power outputs (Liu et al., 2011), (Bejestani et al., 2014). Hence, there is a need for a scheduling framework that would help a VPP to make trading or scheduling decisions, and understand the market risks.

There are several references addressing the optimal operation strategy for DERs in a transactive energy framework. These papers examine different bidding strategies, alternative electricity generation technologies and market structures and optimization approaches (Chen and Hu, 2016),(Wang et al., 2016), (Ghatikar et al., 2016), (Kardakos et al., 2016), (Bejestani et al., 2014).

This paper proposes a hierarchical scheduling framework for a collective form of DERs participating in the hierarchical day-ahead (DA) and real-time (RT) markets, with the transactions among DERs at the distribution level coordinated by the VPP. In the first-stage formulation, the commitment and the hourly scheduling of DERs are determined, helping DERs to maximize the profit in the DA market. In the second stage, the outputs of DERs are optimally adjusted, to minimize the imbalance cost in the RT market. To address risks in relation to uncertainties, the conditional-value-at-risk $(\mathrm{CVaR})$ metric is incorporated into the DA scheduling model. The impacts of risk aversion on VPP profit and system imbalance costs are investigated, and the economic benefits of the proposed approach under different circumstances are discussed.

\section{PROBLEM DESCRIPTION}

\subsection{VPP as a collective form of DERs}

The VPP elements studied include sets of battery energy storage system (BESS) units, gas-fired micro turbines, wind power generators, and end consumers (both interruptible and noninterruptible loads). These DERs are physically interconnected to the upstream distribution networks. The VPP has been granted the authority to participate in markets, acting as a representative that manages all transactions of DERs with the independent system operator (ISO). Thus the VPP is exposed to market risk and needs to optimize its scheduling strategy based on the entire portfolio's operational constraints and the system-state estimation. Moreover, end consumer customers of the VPP are supplied with a given retail energy rate, as a risk insulation strategy. The VPP contracts with interruptible consumers, specifying the upper limit, cost, and permitted hours of load curtailment. Distributed generation (DG) and BESS may be used for trading energy but corresponding costs should be paid. The operational cost of wind power is assumed negligible.

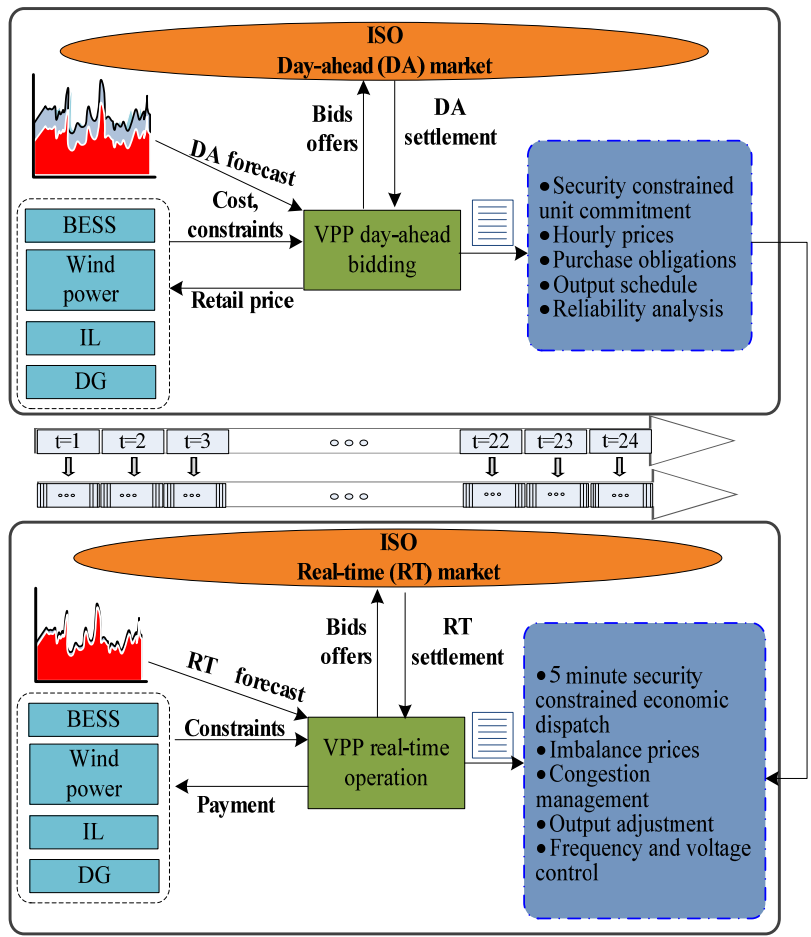

Figure $1 \mathrm{~A}$ two-stage scheduling framework for a VPP.

\subsection{Market information and assumptions}

The market structure assumed here is a joint DA and RT electricity market, and we only focus on energy markets. As seen in Fig. 1, in the DA (spot) market, participants propose their power scheduling for each hour of the coming day before the gate closure (e.g. $12.00 \mathrm{pm}$ ). The interval between the gate closure and the 
beginning of power delivery is at least 12 hours (Chen and Hu, 2016). After the spot price has been settled and commitments have been made, participants are responsible for deviations due to unpredictable fluctuations in power production or consumption. In the RT market, any deviation from the commitment made in the DA market will be settled by a regulation price. If the actual consumption is more than (or the production is less than) the commitment, the power shortage is purchased at an up-regulation price, which is usually higher than the spot price. If the actual consumption is less than (or the production is more than) the commitment, the power surplus is sold at a down-regulation price, which is usually lower than the spot price (Kardakos et al., 2016). Hence, this market structure encourages participants to reduce their forecast errors and be more strategic (Skytte, 1999). All participants, including the VPP, submit operational plans in the form of power stacks.

In the market framework, three assumptions are made for the operation of the VPP. The first assumption is that DERs in the VPP are centrally controlled (Mashhour and Moghaddas-Tafreshi, 2011). This means that the VPP's central controller is responsible for bi-directional communication, monitoring and control of each VPP element. The second assumption is that the VPP can obtain the required data (e.g. price and load), and that relevant time-series forecast techniques are available. The final assumption is that the VPP is a price-taker, which means its scheduling strategy does not influence the market price.

\section{PROPOSED TWO-STAGE SCHEDULING MODEL}

\subsection{Day-ahead (DA) scheduling}

The optimal scheduling model of the VPP in the DA market is formulated as:

$$
\max f_{1}=\left\{\begin{array}{l}
\sum_{t=1}^{T} \rho_{t}^{D A} P_{t}^{D S} \Delta t+\sum_{t=1}^{T} \rho_{t}^{\text {Retail }}\left(P_{D t}-\sum_{i \in \Omega_{D}} P_{i t}^{I L}\right) \Delta t \\
-\sum_{t=1}^{T} C_{t}^{I m b}-\sum_{t=1}^{T} \sum_{i \in \Omega_{D}} C_{i t}^{I L}-\sum_{t=1}^{T} \sum_{i \in \Omega_{B E S S}} C_{i t}^{B E S S} \\
-\sum_{t=1}^{T} \sum_{i \in \Omega_{D G}}\left(C_{i t}^{D G} \chi_{i t}^{D G}+S U C_{i t}^{D G} \chi_{i t}^{S U}+S D C_{i t}^{D G} \chi_{i t}^{S D}\right)
\end{array}\right\}
$$

In (1), the first term represents the cost (revenue) of the VPP by purchasing (selling) electricity from (or to) the distribution system. The second term represents the revenue raised by selling electricity to the customers. The remaining terms in (13) represent the cost of power imbalance and costs paid to IL, BESS and DG.

Given the uncertainties in electricity markets, (1) should be formulated probabilistically. Moreover, the risk associated with the profit variability can be explicitly captured in the model by incorporating the CVaR metric:

$$
E[\operatorname{Pr} \text { ofit }]=\sum_{k \in \Omega_{K}} \operatorname{Pr}_{k} \cdot f_{1 k}+\varpi\left(\operatorname{VaR}-\frac{1}{1-\alpha}\right) \sum_{k \in \Omega_{K}} \operatorname{Pr}_{k} \cdot \psi_{k}
$$

For each scenario, the complete constraints associated with (2) are given below. To avoid clutter, the scenario subscript $k$ is not explicit in the variables below.

1) Supply-demand balance constraint

$$
\sum_{i \in \Omega_{G W}} P_{G W i t}+\sum_{i \in \Omega_{B E S S}} P_{i t}^{B E S S}+\sum_{i \in \Omega_{D G}} P_{D G i t}=P_{D t}-\sum_{i \in \Omega_{D}} P_{i t}^{I L}+P_{t}^{D S}+P_{t}^{\text {Loss }}, \forall t \in 1: T
$$

2) Non-dispatchable wind power constraint

$$
\left\{\begin{array}{l}
0 \leq P_{G W i t} \leq \bar{P}_{G W i} ; \forall i \in \Omega_{G W} \\
P_{G W i t} / \sqrt{P_{G W i t}^{2}+Q_{G W i t}^{2}}=\text { constant }
\end{array}, \forall t \in 1: T\right.
$$

3) Dispatchable DG constraint

$$
\begin{aligned}
& \underline{P}_{D G i} \chi_{i t}^{D G} \leq P_{D G i t} \leq \bar{P}_{D G i} \chi_{i t}^{D G}, \forall t \in 1: T, \forall i \in \Omega_{D G} \\
& \left\{\begin{array}{l}
P_{D G i, t}-P_{D G i, t-1} \leq \operatorname{Ramp}_{D G i}^{U p} \text {, if } P_{D G i, t} \geq P_{D G i, t-1} \\
P_{D G i, t-1}-P_{D G i, t} \leq \operatorname{Ramp}_{D G i}^{D o w n}, \text { if } P_{D G i, t-1} \geq P_{D G i, t}
\end{array}\right. \\
& \left\{\begin{array}{l}
{\left[T_{i, t-1}^{O n}-M U T_{i}\right] \cdot\left[\chi_{i, t-1}^{D G}-\chi_{i t}^{D G}\right] \geq 0} \\
{\left[T_{i, t-1}^{O f f}-M D T_{i}\right] \cdot\left[\chi_{i, t-1}^{D G}-\chi_{i t}^{D G}\right] \geq 0}
\end{array}\right. \\
& \left\{\begin{array}{l}
\chi_{i, t}^{D G}-\chi_{i, t-1}^{D G} \leq \chi_{i t}^{S U} \\
\chi_{i, t-1}^{D G}-\chi_{i, t}^{D G} \leq \chi_{i t}^{S D} \\
\chi_{i, t}^{D G}-\chi_{i, t-1}^{D G} \leq \chi_{i t}^{S U}-\chi_{i t}^{S D}
\end{array}\right.
\end{aligned}
$$


Qiu et al., Scheduling of Distributed Energy Resources as a Virtual Power Plant

4) Interruptible load constraint

$$
\begin{gathered}
\frac{\left|P_{i t}^{I L}\right|}{P_{D i t}} \leq \bar{\varsigma}, \forall t \in 1: T, \forall i \in \Omega_{D} \\
\left\{\begin{array}{l}
\left|P_{i t}^{I L}\right|=P_{i t}^{I L}, \text { if } P_{i t}^{I L} \geq 0 \\
\left|P_{i t}^{I L}\right|=-P_{i t}^{I L}, \text { if } P_{i t}^{I L}<0
\end{array}\right.
\end{gathered}
$$

5) BESS constraint

$$
\begin{aligned}
& E_{i, t+1}^{B E S S}=E_{i, t}^{B E S S}-P_{i t}^{B E S S} \Delta t-\left|P_{i t}^{B E S S}\right| \eta^{C} \Delta t-E_{i, t}^{B E S S} \eta^{L} \Delta t, \forall t \in 1: T, \forall i \in \Omega_{B E S S} \\
& S O C_{i t}=E_{i t}^{B E S S} / E_{R}^{B E S S} \\
& \underline{S O C}_{i t} \leq S O C_{i t} \leq \overline{S O C}_{i t} \\
& \left\{\begin{array}{l}
P_{i t}^{B E S S} \leq \bar{P}_{i}^{\text {BESS,Dis }} \chi_{i t}^{\text {BESS,Dis }} \text {, if } P_{i t}^{\text {BESS }} \geq 0 \\
P_{i t}^{\text {BESS }} \geq \bar{P}_{i}^{\text {BESS,Chr }} \chi_{i t}^{\text {BESS,Chr } r} \text {, if } P_{i t}^{\text {BESS }} \leq 0
\end{array}\right. \\
& \chi_{i t}^{B E S S, D i s}+\chi_{i t}^{B E S S, C h r} \leq 1 \\
& E_{i, 0}^{\text {BESS }}=E_{i, \text { Initial }}^{\text {BESS }} ; E_{i, T}^{\text {BESS }} \geq E_{i, \text { End }}^{\text {BESS }}
\end{aligned}
$$

6) Network constraint

$$
\begin{gathered}
P_{i t}\left(\theta_{t}, V_{t}\right)-P_{G i t}+P_{D i t}=0, \quad \forall t \in 1: T, \forall i \in \Omega_{N} \\
Q_{i t}\left(\theta_{t}, V_{t}\right)-Q_{G i t}+Q_{D i t}=0, \quad \forall t \in 1: T, \forall i \in \Omega_{N} \\
S_{i j t}\left(\theta_{t}, V_{t}\right) \leq \bar{S}_{i j}, \forall t \in 1: T, \forall i, j \in \Omega_{N} \\
\underline{V}_{i} \leq V_{i t} \leq \bar{V}_{i}, \forall t \in 1: T, \forall i \in \Omega_{N}
\end{gathered}
$$

7) Interconnection exchange constraint

$$
\underline{P}^{E x c h} \leq P_{t}^{D S} \leq \bar{P}^{E x c h}, \forall t \in 1: T
$$

Equation (21) states the lower and upper limits of exchange between the VPP and the upstream grid. 8) Steady security reserve constraint

$$
\begin{aligned}
& \sum_{i \in \Omega_{D G}} \bar{P}_{D G i} \chi_{i t}^{D G}-\sum_{i \in \Omega_{D G}} P_{D G i t} \chi_{i t}^{D G}+\sum_{i \in \Omega_{G W}} P_{G W i t} \\
& +\sum_{i \in \Omega_{B E S S}} P_{i t}^{B E S S}+\sum_{i \in \Omega_{D}}\left(\bar{P}_{i t}^{I L}-P_{i t}^{I L}\right) \quad, \forall t \in 1: T \\
& +\left(\bar{P}_{t}^{E x c h}-P_{t}^{D S}\right) \geq P_{D t}-\sum_{i \in \Omega_{D}} P_{i t}^{I L}+R s v(t)
\end{aligned}
$$

Equation (22) states the system reserve requirement $R s v(t)$ for static security and adequacy.

9) CVaR constraint

$$
\begin{gathered}
V a R-f_{1} \leq \psi \\
\psi \geq 0
\end{gathered}
$$

Equations (23) and (24) state the constraint of $\mathrm{CVaR}$ at the $\alpha$-confidence level, i.e., $\alpha-\mathrm{VaR}$

\subsection{Real-time (RT) balancing}

The objective in this stage is to minimize the imbalance cost.

$$
\min f_{2}=\sum_{t=1}^{N T} \hat{C}_{t}^{I m b}
$$

We simply use $(\hat{\bullet})$ to distinguish the variables in the second stage from the variables in the first stage.

$$
\begin{gathered}
\Delta \hat{P}_{t}^{\operatorname{Im} b}=\hat{P}_{t}^{R T}-P_{t}^{D S} \\
\hat{P}_{t}^{R T}=\sum_{i \in \Omega_{G W}} \hat{P}_{G W i t}+\sum_{i \in \Omega_{B E S S}} \hat{P}_{i t}^{B E S S}+\sum_{i \in \Omega_{D G}} \hat{P}_{D G i t}-\left(\hat{P}_{D t}+\hat{P}_{t}^{\text {Loss }}-\sum_{i \in \Omega_{D}} \hat{P}_{i t}^{I L}\right) \\
\hat{C}_{t}^{I m b}=\left\{\begin{array}{l}
\rho_{t}^{R+} \cdot \Delta \hat{P}_{t}^{I m b}, \Delta P_{t}^{I m b}<0 \\
\rho_{t}^{R-} \cdot \Delta \hat{P}_{t}^{I m b}, \Delta P_{t}^{I m b} \geq 0
\end{array}\right.
\end{gathered}
$$

The complete constraints of (25) include (3)-(6), (9)-(14), and (16)-(22). 


\section{CASE STUDIES}

\subsection{5-bus VPP}

The proposed scheduling approach is tested on a 15-bus VPP model. The system comprises four wind turbines, three BESS, three DG units, and 13 load buses. The total generation capacity is $8100 \mathrm{~kW}$, including $2700 \mathrm{~kW}$ of wind power and $5400 \mathrm{~kW}$ of DG. We assume that IL is located at every load bus, and up to $20 \%$ of the system load can be adjusted if necessary. The cost coefficients of IL $a_{1 i}^{I L}$ and $a_{2 i}^{I L}$ are set at $\$ 0.01 / \mathrm{MW}^{2} \mathrm{~h}$ and $\$ 0.5 / \mathrm{MWh}$ respectively, based on the IL cost in ( $\mathrm{Li}$ et al., 2015). The capacity of power interconnection between VPP and the main grid is set as $2400 \mathrm{~kW}$. For simplicity, the minimum up time and down time of DG are assumed to be 3 hours and 2 hours respectively. Also, the start-up and shut-down costs are assumed to be $\$ 70$ and $\$ 20$ respectively.

Three operation strategies are compared. Mode 1 is an uncoordinated operation strategy. An individual VPP element operates strategically as a price-taker in the DA market, aiming to maximize its profits. The total profit is the sum of individual's profit virtually obtained. The imbalance cost is calculated after the realization of scenarios. Mode 2 is a coordinated operational strategy by a single VPP entity, but without the RT balancing problem in Section 3.2. Mode 3 is the proposed VPP scheduling strategy. The confidence level $\alpha$ is set at $90 \%$ and the variance adjustment
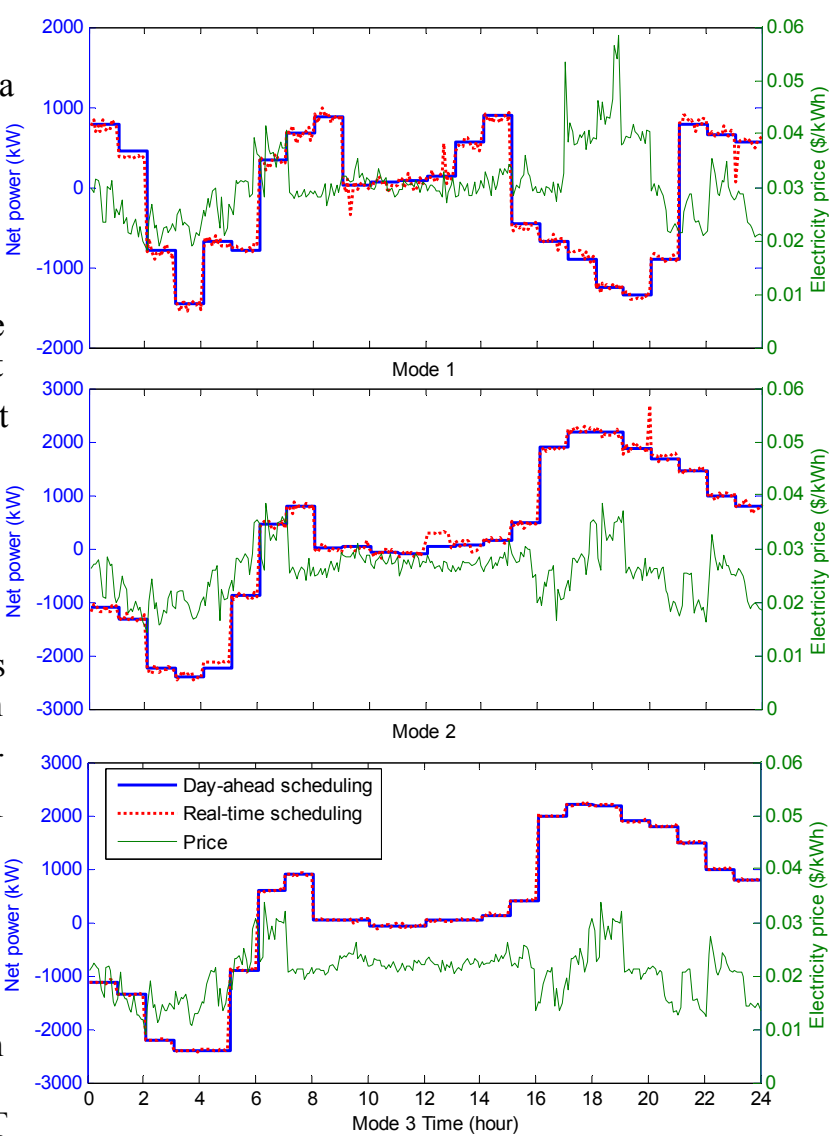

Figure 2. Exchanged power in the DA and RT markets and energy prices for operation mod weighting factor $\Phi$ in the objective is set at 0.8 . Thus CVaR denotes the risk adjusted expected value of the $10 \%$ scenarios with lowest profit. As illustrated in Fig. 2, the results show that the VPP prefers to buy electricity from the main grid in the early morning (3-5 am): limited power exchange occurs between 10 am and $12 \mathrm{pm}$ in all three operation modes. In mode 1, the largest import is $1456 \mathrm{~kW}$ at $4 \mathrm{am}$, and the largest export is 890 $\mathrm{kW}$ at $3 \mathrm{pm}$. In mode 2, the largest import is $2398 \mathrm{~kW}$ at $4 \mathrm{am}$, and the largest export is $2190 \mathrm{~kW}$ at $6 \mathrm{pm}$. In mode 3, the largest import is $2400 \mathrm{~kW}$ at $4 \mathrm{am}$, and the largest export is $2205 \mathrm{~kW}$ at $6 \mathrm{pm}$. Without coordination, the VPP elements need energy from the grid to satisfy its peak demand (i.e. negative power exchange between $6 \mathrm{pm}$ and $8 \mathrm{pm}$ ). By contrast, a coordinated VPP (modes 2 and 3 ) is able to sell electricity in peak hours. This means that a coordinated VPP uses its DG, IL and BESS efficiently to make profits, by selling electricity when the market price is high. Generally, the deviations between the DA and RT markets are the largest in mode 1, whereas deviations are the smallest in mode 3. Thus, the coordinated two-stage VPP scheduling is more accurate and incurs less imbalance cost. Also, mode 1 faces the highest electricity cost, and the price spike in peak hours can reach about $\$ 0.058 / \mathrm{kWh}$. The average prices for modes 1 to 3 are $\$ 0.035, \$ 0.029$ and $\$ 0.024 / \mathrm{kWh}$ respectively. This reveals that the proposed approach incurs the lowest energy price.

In the base case, for mode 3 the expected profit is $\$ 7.84 \mathrm{~K}, \mathrm{CVaR}$ is $\$ 3.76 \mathrm{~K}$, the imbalance cost is $-\$ 1.15 \mathrm{~K}$, the energy traded in the DA market is $26.05 \mathrm{MWh}$, the energy traded in the RT market is $364.49 \mathrm{kWh}$ and the total actual DG production is $52.78 \mathrm{MWh}$. Four cases are used to conduct sensitivity analysis: in Case 1 wind power penetration increases by 10\%, in Case 2 load forecast error increases by $10 \%$, in Case 3 the imbalance price increases by $10 \%$ and in Case 4 there is no risk aversion.

Several observations are made in Table I. First, the increased wind power results in higher profits in the DA market, incurs a greater imbalance cost and reduces DG production. Wind power poses significant risk to the VPP, and more energy is traded in the DA and RT markets. This risk can be effectively mitigated by the coordination of the VPP elements (e.g. imbalance cost is reduced from $\$ 4.52 \mathrm{~K}$ in mode 1 to $\$ 1.98 \mathrm{~K}$ and $\$ 1.35 \mathrm{~K}$ in modes 2 and 3, respectively). Second, the increased load forecast errors have little impact on the expected profit, the energy traded in the DA market and DG production, but the VPP is likely to incur a higher imbalance cost. Third, the increased imbalance price makes the VPP 20\% more conservative, in the sense that the energy traded in the DA market increases from 26.05 MWh to 31.25 MWh in mode 3. Also, the VPP uses about $18 \%$ 
more DG (production increases from 52.78 MWh to 62.35 MWh in mode 3) and is more willing to import power from the grid, rather than relying on wind power. Fourth, without risk aversion, the VPP becomes less conservative. For instance in mode 3, compared to the base case, the energy traded in the RT market increases from $364.49 \mathrm{kWh}$ to $439.21 \mathrm{kWh}$.

Table 1. Sensitivity Analysis of Different Cases (Note $\$ K=\$ 1000)$

\begin{tabular}{|c|c|c|c|c|c|c|c|}
\hline 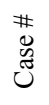 & $\sum^{\frac{0}{0}} \#$ & $\begin{array}{l}\text { Expected } \\
\text { Profit (\$K) }\end{array}$ & $\begin{array}{c}\mathrm{CVaR} \\
(\$ \mathrm{~K})\end{array}$ & $\begin{array}{l}\text { Imbalance } \\
\text { Cost (\$K) }\end{array}$ & $\begin{array}{c}\text { Energy traded } \\
\text { in DA } \\
\text { (MWh) }\end{array}$ & $\begin{array}{c}\text { Energy traded } \\
\text { in RT } \\
(\mathrm{kWh})\end{array}$ & $\begin{array}{l}\text { DG production } \\
\text { (MWh) }\end{array}$ \\
\hline 2 & $\begin{array}{l}1 \\
2 \\
3 \\
\end{array}$ & $\begin{array}{l}6.98 \\
7.16 \\
7.84 \\
\end{array}$ & $\begin{array}{l}3.18 \\
3.32 \\
3.56 \\
\end{array}$ & $\begin{array}{l}-2.03 \\
-1.94 \\
-1.31 \\
\end{array}$ & $\begin{array}{l}22.36 \\
27.13 \\
26.03 \\
\end{array}$ & $\begin{array}{l}615.36 \\
601.78 \\
418.68 \\
\end{array}$ & $\begin{array}{l}62.36 \\
53.21 \\
52.78 \\
\end{array}$ \\
\hline 3 & $\begin{array}{l}1 \\
2 \\
3 \\
\end{array}$ & $\begin{array}{l}7.03 \\
7.89 \\
8.15 \\
\end{array}$ & $\begin{array}{l}3.12 \\
3.58 \\
4.08 \\
\end{array}$ & $\begin{array}{l}-2.15 \\
-2.01 \\
-1.45 \\
\end{array}$ & $\begin{array}{l}24.58 \\
31.29 \\
31.25 \\
\end{array}$ & $\begin{array}{l}412.36 \\
312.85 \\
265.31 \\
\end{array}$ & $\begin{array}{l}71.32 \\
63.25 \\
62.35 \\
\end{array}$ \\
\hline 4 & $\begin{array}{l}1 \\
2 \\
3\end{array}$ & $\begin{array}{l}7.11 \\
7.96 \\
7.93 \\
\end{array}$ & $\begin{array}{l}2.96 \\
3.02 \\
3.51 \\
\end{array}$ & $\begin{array}{l}-3.05 \\
-2.15 \\
-1.89 \\
\end{array}$ & $\begin{array}{l}23.84 \\
31.55 \\
33.25 \\
\end{array}$ & $\begin{array}{l}613.31 \\
596.78 \\
439.21 \\
\end{array}$ & $\begin{array}{l}60.31 \\
59.25 \\
45.82 \\
\end{array}$ \\
\hline
\end{tabular}

\section{CONCLUSION}

This paper presents a scheduling model of DERs within a transactive energy framework. To provide sensitivity to profit variability, the CVaR metric is incorporated into the objective function. Thus, the VPP can flexibly adjust its scheduling strategy. Simulation results show that if a more conservative strategy is adopted, the willingness to use DG and to buy electricity from the grid increases, hence, less energy is traded in the RT market. Furthermore, coordinated scheduling can effectively offset the wind power fluctuation and mitigate the impacts of uncertainties. With the two-level scheduling, the risk exposure can be mitigated, and the cost component related to the risk aversion in the objective is also effectively reduced. To sum up, coordinated twolevel scheduling is a flexible risk-hedging tool that can identify optimal and accurate operation plans.

\section{REFERENCES}

Asmus, P. (Nov. 2010). 'Microgrids, virtual power plants and our distributed energy future', Electr. J., vol. 23(10), pp. 72-82.

Bejestani, A. K., A. Annaswamy and T. Samad (Jul. 2014). 'A hierarchical transactive control architecture for renewables integration in smart grids: analytical modeling and stability', IEEE Trans. Smart Grid, vol. 5(4), pp. 2054-2065.

Chen, Y. and M. Hu (Aug. 2016). 'Balancing collective and individual interests in transactive energy management of interconnected micro-grid clusters', Energy, vol. 109, pp. 1075-1085.

Ghatikar, G., S. Mashayekh, M. Stadler, R. Yin and Z. Liu (April 2016). 'Distributed energy systems integration and demand optimization for autonomous operations and electric grid transactions', Appl. Energy, vol. 167, pp. 432-448.

Kardakos, E. G., C. K. Simoglou and A. G. Bakirtzis (Mar. 2016). 'Optimal offering strategy of a virtual power plant: a stochastic bi-level approach', IEEE Trans. Smart Grid, vol. 7(2), pp. 794-806.

Li, C. X., Z. Y. Dong, G. Chen, F. J. Luo and J. Liu (2015). 'Flexible transmission expansion planning associated with large-scale wind farms integration considering demand response', IET Gen. Trans. \& Dist., vol. 9(15), pp. 2276-2283.

Liu, Z., F. Wen and G. Ledwich (Oct. 2011). 'Optimal siting and sizing of distributed generators in distribution system considering uncertainties', IEEE Trans. Power Deliv., vol. 26(4), pp. 2541-2551.

Mashhour, E. and S. M. Moghaddas-Tafreshi (May 2011). 'Bidding strategy of virtual power plant for participating in energy and spinning reserve markets-part I: problem formulation', IEEE Trans. Power Syst., vol. 26(2), pp. 949-956.

Pandzic, H., J. M. Morales, A. J. Conejo and I. Kuzle (May 2013). 'Offering model for a virtual power plant based on stochastic programming', Appl. Energy, vol. 105, pp. 282-292.

Skytte, K. (1999). 'The regulating power market on the Nordic power exchange Nord Pool: an econometric analysis', Energy Econom., vol. 21(4), pp. 295-308.

Wang, Y., X. Ai, Z. Tan, L. Yan and S. Liu (Jan. 2016). 'Interactive dispatch modes and bidding strategy of multiple virtual power plants based on demand response and game theory', IEEE Trans. Power Syst., vol. 7(1), pp. 510-519. 